\title{
A Fuzzy MCDM Approach for Evaluating Corporate Image and Reputation in the Airline Market
}

\author{
Mei-Ling Chuang* \\ Department of Transportation and Communication, \\ Taoyuan Country, Taiwan \\ E-mail:mooichuang@hotmail.com \\ Wei-Mao Chen \\ Department of Air Transportation, \\ Kainan University, \\ No. 1 Kainan Road Luzhu, \\ Taoyuan County 338, Taiwan \\ E-mail: $\underline{\text { m96127004@ms.knu.edu.tw }}$ \\ James J.H. Liou \\ Department of Air Transportation, \\ Kainan University, \\ No. 1 Kainan Road Luzhu, \\ Taoyuan County 338, Taiwan \\ E-mail:jhliou@mail.knu.edu.tw
}

\begin{abstract}
In today's highly competitive airline market, having a preferable corporate image is acknowledged as having a positive impact customer loyalty. Corporate image is a powerful way to differentiate a company from its competitors and to stimulate purchasing. In the past, corporate image was a vague concept, difficult to measure quantitatively. Here we propose a fuzzy Multi Criteria Decision Making (MCDM) model which can quantify corporate image and reputation, thus making it easier for management to fully comprehend the relative position of their company in the overall market, and helping them to make informed judgments for the formation of marketing strategies. A study of international airlines serving Taiwan is conducted for verification. Two factors, safety record and service, emerge as critical in the air transport market, while marketing incentives seem to have little attraction for customers.
\end{abstract}

Keywords: corporate image; fuzzy integral; factor analysis; Analytical Hierarchy Process; AHP;

${ }^{*}$ Corresponding author 
Multiple Criteria Decision-Making; MCDM.

\section{Introduction}

Corporate image is a mental picture of a company held by an audience-what comes to mind when one sees or hears the corporate name or sees its logo. Corporate image and reputation have in the past been perceived as rather vague concepts, at best of peripheral concern to senior management. Typically, they were seen as in the domain of the public relations department. Today, however, an increasing number of astute executives recognize that corporate image and reputation are critical corporate assets directly linked to competitive success [Gray and Balmer, 1998]. Southwest Airlines, for instance, in addition to having a visible profile in the airline industry, has an admirable world-wide reputation for low cost and an excellent safety record, that have become the benchmark of a successful air transport business. Companies in the service industry view themselves as in the business of creating outstanding services/experiences for their customers. In most cases, however, high quality, luxury ornamentation, excellent cuisine, comfortable amenities, and good service alone are no longer sufficient to secure a competitive advantage [Campbell and Verbeke, 1994]. Corporate image can sometimes provide a powerful signal differentiating a company from its competitors and stimulating purchases. It is also widely believed that a favorable reputation among employees can be a prime causal factor of high morale and productivity [Gray and Balmer, 1998]. However, the management of corporate image is a challenging task, particularly in the service industry, where products are essentially intangible [Nguyen and Leblanc, 2001]. If managed properly, a positive corporate image can add value to a firm in a variety ways. On the other hand, a negative image can destroy a firm's reputation and alienate their customers.

Traditionally, managers have been more comfortable dealing with quantitative data than consideration of corporate image. Corporate image is unfamiliar, intangible, subjective, transitory, and even personal. As a result, corporate image management-if attempted at all—is often performed in a limited and uncoordinated fashion. Image-related decisions are made in isolated and piecemeal fashion, with little effort and ability brought to bear on formulating an organized, strategic approach [Schmitt et al., 1995]. Moreover, because corporate image is considered the global outcome of processes of legitimization [Rao, 1994], its level of abstraction may be high; consequently, its conceptualization is complex and difficult to measure. A review of the research to date reveals few quantitative models regarding corporate image. With this in mind, the objective of this paper is to develop a fuzzy Multi Criteria Decision Making (MCDM) model with a set of decision criteria for the formation of a corporate image management system. The fuzzy MCDM approach can be used enhance the firm's position by quantitatively measuring its image and reputation in relation to its competitors, thereby helping management to develop better strategies. The proposed fuzzy MCDM model is utilized to identify the criteria for corporate image, which are classified into different aspects. The criteria are weighted and performance quantified, so that management can fully comprehend their company's relative position in the market and formulate better marketing strategies. The feasibility of 
this model is demonstrated by conducting an empirical study of international airlines.

\section{Constructing The Evaluation System For Corporate Image}

Without proper identification of the elements related to corporate image, the comprehensiveness and systematization of strategic qualities will be compromised [Schmitt et al., 1995]. Corporate image can be described as the overall impression made by a firm on the minds of the public [Barich and Kotler, 1991; Dichter, 1985]. It is related to the various physical and behavioral attributes of that firm, such as the business name, architecture, variety of products/services, tradition, ideology, and the impression of quality communicated by each person interacting with the firm's clients [Nguyen and Leblanc, 2001]. Schmitt et al. (1995) suggested that the image of a firm includes the four Ps: property, products, presentation, and publications. Property includes the physical assets of that firm, such as buildings, vehicles, and so on. Products include the goods that a company produces or the core service that it performs. Presentation includes the immediate objects that 'represent' the product, whether an item or a service. Finally, publications which can include printed and broadcast information emanating from the company. Gray and Balmer [1998] describe corporate image as a mental picture of a company, but they do not identify the essential elements. In the service industry, corporate image is viewed as being similar to brand recognition and it is more important than in other industries that produce tangible products. Thus it can be seen that the elements that make up corporate image are complex, and that corporate image is a subjective perception by the customer audience. There is no universal agreement among researchers and practitioners about what exactly constitutes corporate image.

It is very difficult for a company to see its corporate image as its competitors do, or to define clear elements/criteria to quantitatively measure its reputation. This is especially true of the service industry, where corporate image is highly subjective, abstract and culture-dependent. Keeney and Raiffa [1976] suggest that there are five principles that need to be considered when formulating criteria: completeness (the criteria must embrace all of the important characteristics of the decision-making problems), operational (the criteria have to be meaningful to the decision-makers and available for open study), decomposable (the criteria can be decomposed and arranged hierarchically from higher to lower so that the evaluation processes can be simplified), non-redundancy (the criteria must avoid duplicate measurement of the same performance), and minimum size (the number of criteria should be as small as possible so as to reduce the needed manpower, time, and cost). To identify these aspects properly, it is useful for a manager to think of what elements are related to their corporate image. Since customer impression is shaped by culture, an evaluation system in one country may not fit in another country. For example, Tokyo Disneyland has been a huge success, while the Euro Disney theme park has not. One French critic has ridiculed it as 'a horror of cardboard, plastic and appalling colors' [Schmitt et al., 1995]. This confirms that some elements of corporate image are culture-dependent and we need to be properly selected to reflect both operational environment and local diversity. In this study, we use the airline industry as an example, but certainly similar procedures can be used in the other industries. It is worth noting that each market will have its own set 
of criteria and hierarchical structure, because of different cultures and customer needs. We attempt here to present a generalized model based on factors and criteria taken from the literature and survey data. This model can then be adapted or extended for each particular situation.

\section{Evaluation Methods For Corporate Image}

In this next section we establish a mathematical model for quantitative evaluation of the corporate image. The section is divided into three subsections: weighting the evaluation criteria, the application of fuzzy set theory, and acquiring and assessing the performance value [Hsieh et al., 2004; Chiou et al., 2005; Hsu et al., 2005].

\subsection{Determining the Evaluation Criteria Weights}

Because the criteria of corporate image entail diverse significance and meaning, we cannot assume that each considered criteria is of equal importance. There are many methods that can be employed to determine their weights [Hwang and Yoon, 1981], such as the eigenvector method, weighted least squares method, entropy method, Analytic Hierarchy Process (AHP), as well as linear programming techniques for multidimensional analysis. The method selected depends on the nature of the problem. Since corporate image is both complex and vague, the solution requires one to use the most inclusive and flexible method. Here we use the fuzzy AHP approach to determine the criteria weights, because it makes the systematization of complicated problems easy, and allows for the integration of expert and evaluator opinions.

AHP was first proposed by Saaty in 1971 [Saaty, 1977, 1980], and is now widely used in many fields, such as for economic planning, choosing policies, portfolio selection, and several areas of social management sciences. In AHP the principal eigenvector of the pair-wise comparison matrix contrived by a scaling ratio is used to find the comparative weight among the criteria of the hierarchy. Buckley [1985a] further extended Saaty's AHP to cases where evaluators employ fuzzy ratios in place of exact ratios when comparing two criteria, and derive fuzzy weights for the criteria by the geometric mean method. In this study, we employ Buckley's method, fuzzy AHP, to 'fuzzify' hierarchical analysis by replacing pair-wise comparisons with fuzzy numbers to find the fuzzy weights.

Suppose there is a set of $n$ criteria for pairs according to their relative weight scaling. Denote the criteria by $c_{1}, c_{2}, \ldots, c_{n}$ and their weight weights by $w_{1}, w_{2}, \ldots, w_{n}$. If $\boldsymbol{w}=\left(w_{1}, w_{2}, \ldots, w_{n}\right)^{t}$ is given, then matrix $\boldsymbol{A}$ represents the pair-wise comparisons,

$$
\left(\boldsymbol{A}-\lambda_{\max } I\right) \boldsymbol{w}=0 .
$$

Now we find the eigenvector $\boldsymbol{w}$ with the $\lambda_{\max }$ that satisfies $A \boldsymbol{w}=\lambda_{\max } \boldsymbol{w}$. Since the relative importance comparison is divided by intuitive judgment, a certain degree of inconsistency exists. Saaty [1980] used the consistency index (CI) as an indicator of 'closeness to consistency,' $\mathrm{CI}=\left(\lambda_{\max }-n\right) /(n-1)$. In general, the value of $\lambda_{\max }$ can be accepted if CI is not greater than 0.1.

\subsection{The Fuzzy Analytic Hierarchy Process (FAHP)}

Since Zadeh [1965] first introduced fuzzy set theory and subsequently the fuzzy decision-makng method [Bellman and Zadeh, 1970] in fuzzy environments, many other studies have been done where 
fuzzy set theory has been applied to uncertain fuzzy problems. The fuzzy approach has been found suitable to obtain the performance value. The preferable values for corporate image are usually based on vague subjective judgments. The application of fuzzy theory is described below.

\subsection{Fuzzy Number}

According to the definition of Dubois and Prades [1978], the fuzzy number $\tilde{A}$ is a fuzzy set, and its membership function is $u_{\tilde{A}}(x): R \rightarrow[0,1]$. It is common to use triangular fuzzy numbers (TFNs)

$u_{\tilde{A}}(x)=\left(a_{1}, a_{2}, a_{3}\right)$ as shown in Eq. (2)

$$
\mu_{\tilde{A}}(x)=\left[\begin{array}{cl}
\left(x-a_{1}\right) /\left(a_{2}-a_{1}\right), & a_{1} \leq x \leq a_{2}, \\
\left(a_{3}-x\right) /\left(a_{3}-a_{2}\right), & a_{2} \leq x \leq a_{3}, \\
0, & \text { otherwise, }
\end{array}\right.
$$

where $a_{1}$ and $a_{3}$ stand for the lower and upper bounds of the fuzzy number $\tilde{A}$, respectively; and $a_{2}$ stands for the modal value. The operational laws of two TFNs $\tilde{A}=\left(a_{1}, a_{2}, a_{3}\right)$ and $\widetilde{B}=\left(b_{1}, b_{2}, b_{3}\right)$ are as follows:

Addition of two fuzzy numbers $\oplus$,

$$
\left(a_{1}, a_{2}, a_{3}\right) \oplus\left(b_{1}, b_{2}, b_{3}\right)=\left(a_{1}+b_{1}, a_{2}+b_{2}, a_{3}+b_{3}\right) ;
$$

Subtraction of two fuzzy numbers $\Theta$,

$$
\left(a_{1}, a_{2}, a_{3}\right) \Theta\left(b_{1}, b_{2}, b_{3}\right)=\left(a_{1}-b_{3}, a_{2}-b_{2}, a_{3}-b_{1}\right) ;
$$

Multiplication of two fuzzy numbers $\otimes$,

$$
\left(a_{1}, a_{2}, a_{3}\right) \otimes\left(b_{1}, b_{2}, b_{3}\right) \cong\left(a_{1} b_{1}, a_{2} b_{2}, a_{3} b_{3}\right) ;
$$

Division of two fuzzy number $\nabla$,

$$
\left(a_{1}, a_{2}, a_{3}\right) \nabla\left(b_{1}, b_{2}, b_{3}\right)=\left(a_{1} / b_{3}, a_{2} / b_{2}, a_{3} / b_{1}\right) .
$$

\subsection{Linguistic Variables}

According to Zadeh [1975], it is very difficult during conventional quantification to reasonably express situations that are overtly complex or hard to define; the notion of a linguistic variable is necessary in such situations. A linguistic variable is a variable whose values are words or sentences in a natural or artificial language. Here, we use this kind of expression to compare two corporate image criteria. We use five basic linguistic terms: 'absolutely important,' 'very strong important,' 'essentially important,' 'weakly important,' 'equally important' with respect to fuzzy numbers as defined by Mon et al. [1994] in Table 1. Similarly, the linguistic variables used for the criteria for the corporate image performance value are: 'very good,' 'good,' 'fair,' 'poor,' 'very poor.' The membership functions of the expression values can be indicated by triangular fuzzy numbers, as shown in Figure 1.

Table 1.Membership Function For The Linguistic Scale 


\begin{tabular}{cll}
\hline$\tilde{1}$ & Equally important (Eq) & $(1,1,3)$ \\
$\tilde{3}$ & Weakly important (Wk) & $(1,3,5)$ \\
$\tilde{5}$ & Essentially important (Es) & $(3,5,7)$ \\
$\tilde{7}$ & Very strongly important (Vs) & $(5,7,9)$ \\
$\tilde{9}$ & Absolutely important (Ab) & $(7,9,9)$ \\
\hline
\end{tabular}

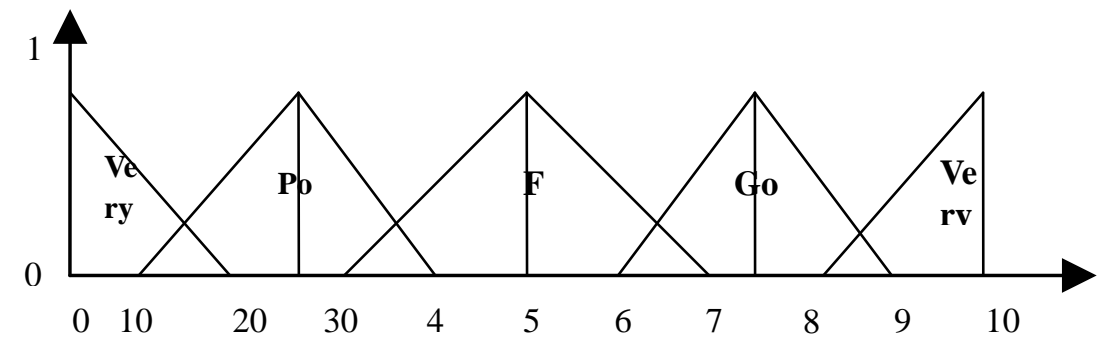

Figure 1.Example Of The Membership Function For The Linguistic Variables For Measuring The Performance Values Of Alternatives

\subsection{Procedures of the Fuzzy Analytic Hierarchy Process}

The procedure for determining the weights of evaluating criteria by fuzzy AHP are summarized as follows:

Step 1. Construct pair-wise comparison matrices from among all elements/criteria of the hierarchy. Assign linguistic terms to the pair-wise comparisons by asking which is the more important of each of two elements/criteria.

Step 2. Use the geometric mean technique to define the fuzzy geometric mean and fuzzy weights for each criterion, as done by Buckley [1985b]

$$
\begin{aligned}
& \tilde{r}_{i}=\left(\tilde{a}_{i 1} \otimes \tilde{a}_{i 2} \otimes \cdots \otimes \tilde{a}_{i n}\right)^{1 / n}, \\
& \tilde{w}_{i}=\tilde{r}_{i} \otimes\left(\tilde{r}_{i} \oplus \cdots \oplus \tilde{r}_{n}\right)^{-1},
\end{aligned}
$$

where $\tilde{a}_{i n}$ is the fuzzy comparison value of criterion $i$ to criterion $n$. Thus, $\tilde{r}_{i}$ is the geometric mean of the fuzzy comparison value of criterion $i$ for each criterion; and $\tilde{w}_{i}$ is the fuzzy weight of the $i^{\text {th }}$ criterion, which can be expressed by a TFN.

\subsection{Fuzzy Multiple Criteria Decision-Making (FMCDM)}

In this study fuzzy MCDM is used to evaluate the performance value of corporate image and to rank 
them according to priority. The fuzzy MCDM procedure is described below:

Step 1. Measuring criteria: use the measurement of linguistic variables to estimate the criteria performance. The evaluators are asked their subjective judgment; each linguistic variable can be indicated by a TFN within the scale range $0-100$, as shown in Figure 1 . Let $\widetilde{E}_{i j}^{k}$ be the fuzzy performance value of the $k^{\text {th }}$ evaluator toward corporate image $i$ under criterion $j$; all of the evaluation criteria will be indicated by a TFN as in Eq (8)

$$
\tilde{E}_{i j}^{k}=\left(L \tilde{E}_{i j}^{k}, M \tilde{E}_{i j}^{k}, U \tilde{E}_{i j}^{k}\right)
$$

Since the perception of each evaluator varies according to their knowledge and experience, the definitions of the linguistic variable will vary as well. Here we use the notation of the average value to integrate the fuzzy judgment values of $m$ evaluators, that is,

$$
\widetilde{E}_{i j}=(1 / m) \otimes\left(\tilde{E}_{i j}^{1} \oplus \tilde{E}_{i j}^{2} \oplus \cdots \oplus \tilde{E}_{i j}^{m}\right),
$$

where $\otimes$ denotes fuzzy multiplication; $\oplus$ denotes fuzzy addition; $\widetilde{E}_{i j}$ is the average fuzzy number of the judgment of $m$ evaluators; and a TFN is shown as

$$
\widetilde{E}_{i j}=\left(L \widetilde{E}_{i j}, M \tilde{E}_{i j}, U \tilde{E}_{i j}\right) .
$$

The end-point values $L E_{i j}, M E_{\mathrm{ij}}$, and $U E_{\mathrm{ij}}$ can be solved by

$$
\begin{aligned}
L E_{i j} & =\left(\sum_{k=1}^{m} L E_{i j}^{k}\right) / m ; M E=\left(\sum_{k=1}^{m} M E_{i j}^{k}\right) / m ; \\
U E_{i j} & =\left(\sum_{k=1}^{m} U E_{i j}^{k}\right) / m .
\end{aligned}
$$

Step 2. Fuzzy synthetic decision: the weights of the criteria and fuzzy performance values must be integrated by the fuzzy number operation, so as to calculate the fuzzy performance value of the integral evaluation for each alternative. According to the fuzzy AHP method, we get a weight vector $\widetilde{w}=\left(\tilde{w}_{1}, \widetilde{w}_{2} \ldots, \widetilde{w}_{n}\right)^{t}$. The fuzzy performance matrix $\tilde{E}$ for each the corporate image can now be obtained from Eq. (9) under $n$ criteria. From the criteria weight vector $\widetilde{w}$ and the fuzzy performance matrix $\tilde{E}$, the final fuzzy synthetic decision can be conducted. The derived results will be the fuzzy synthetic decision matrix $\stackrel{\widetilde{R}}{R}$, that is,

$$
\widetilde{R}=\widetilde{E} * \widetilde{w}
$$

where “*” indicates the calculation of the fuzzy numbers, including fuzzy addition and fuzzy multiplication. Because the operation of fuzzy multiplication is relatively complex, it is usually denoted by an approximate multiplied result $\widetilde{R}=\left(L R_{i}, M R_{i}, U R_{i}\right)$, where $L R_{i}, M R_{i}$, and $U R_{i}$ are the lower, middle and upper synthetic performance values of the alternative $i$. It can be expressed as follows: 


$$
\begin{aligned}
& L R_{i}=\sum_{j=1}^{n} L E_{i j} \times L w_{j} ; \quad M R_{i}=\sum_{j=1}^{n} M E_{i j} \times M w_{j} ; \\
& U R_{i}=\sum_{j=1}^{n} U E_{i j} \times U w_{j} .
\end{aligned}
$$

Step 3. Comparison of the corporate image: the result of the fuzzy synthetic decision reached by each alternative is a fuzzy number. It is necessary to transform the fuzzy number into a non-fuzzy number in order to compare them. In many studies the procedure used for defuzzification has been to locate the best non-fuzzy performance (BNP) value. Such defuzzified fuzzy ranking methods generally include the mean of maximal (MOM), center of area (COA), and $\alpha$-Cut [Zhao and Goving, 1991; Teng and Tzeng, 1996]. Utilizing the COA method to determine the BNP is simple and practical. The BNP value of the fuzzy number can be calculated as follows:

$$
B N P_{\mathrm{i}}=\left[\left(U R_{\mathrm{i}}-L R_{\mathrm{i}}\right)+\left(M R_{\mathrm{i}}-L R_{\mathrm{i}}\right)\right] / 3+L R_{\mathrm{i}} \forall i .
$$

After the value of the BNP has been derived for each alternative, we can then proceed with the ranking of the corporate image alternatives. In the next section, the airline industry is used as an empirical example to validate the model.

\section{Empirical Study Of Corporate Image In The Airline Market}

To illustrate the effectiveness of the fuzzy MCDM approach presented above, we examine eight international airlines serving in Taiwan. As suggested by Keeney and Raiffa [1976], the complex evaluation environment can be divided into many criteria or subsystems. It is easier to judge differences and to measure scores for divided criteria or subsystems. The fuzzy AHP method is used to determine the weights of the various aspects and criteria. The performance of the selected airlines is evaluated according to those criteria. Finally, the simple additive-weight method is used to decide the relative ranking of the alternatives. The model allows airlines to evaluate their image and reputation level, and to develop strategies for further expanding their market-share.

\subsection{Evaluating the Hierarchical System and its Criteria}

We first selected 35 airline image related elements via consultation with airline public-relation department managers and through a literature review [Nguyen and Leblanc, 2001; Ruyter and Wetzels, 2000; Gray and Balmer, 1998; Williams and Moffitt, 1997; Schmitt et al., 1995]. These 35 items included the firm's physical assets (e.g. buildings and aircraft), products (e.g. comfort of seats and service at each stage), presentation (e.g. clothing, logo and decorations), performance (e.g. on-time performance and safety record) and publications (e.g. advertisements, brochures), morality (e.g. participation in charity activities), and management (e.g. management style, employee skills) etc. In a questionnaire survey, 52 interviewees, including tour leaders, airline public-relations department employees, and customers from different backgrounds, were asked questions. Respondents were asked to rate the importance of 35 element related to airline image on a 5-point scale ranging from 5 (extremely important) to 0 (no effect). They could also add items that they thought important to airline 
image. The criteria with the highest aggregate score for the 16 elements were selected to construct the evaluating system. The 16 criteria were divided into the following five categories: morality, management, economics, service, and convenience. Morality included the safety record and participation in charitable activities. Management included management style, employee skills, teamwork, and training. Economics included reasonability of prices, promotional activities, and sales channels. Service included the handling of complaints, focusing on customer interests, comfort of the seats, and cabin crew service. Convenience included scheduling, on-time performance, and routes. The hierarchical system for airline image is shown in Figure 2.

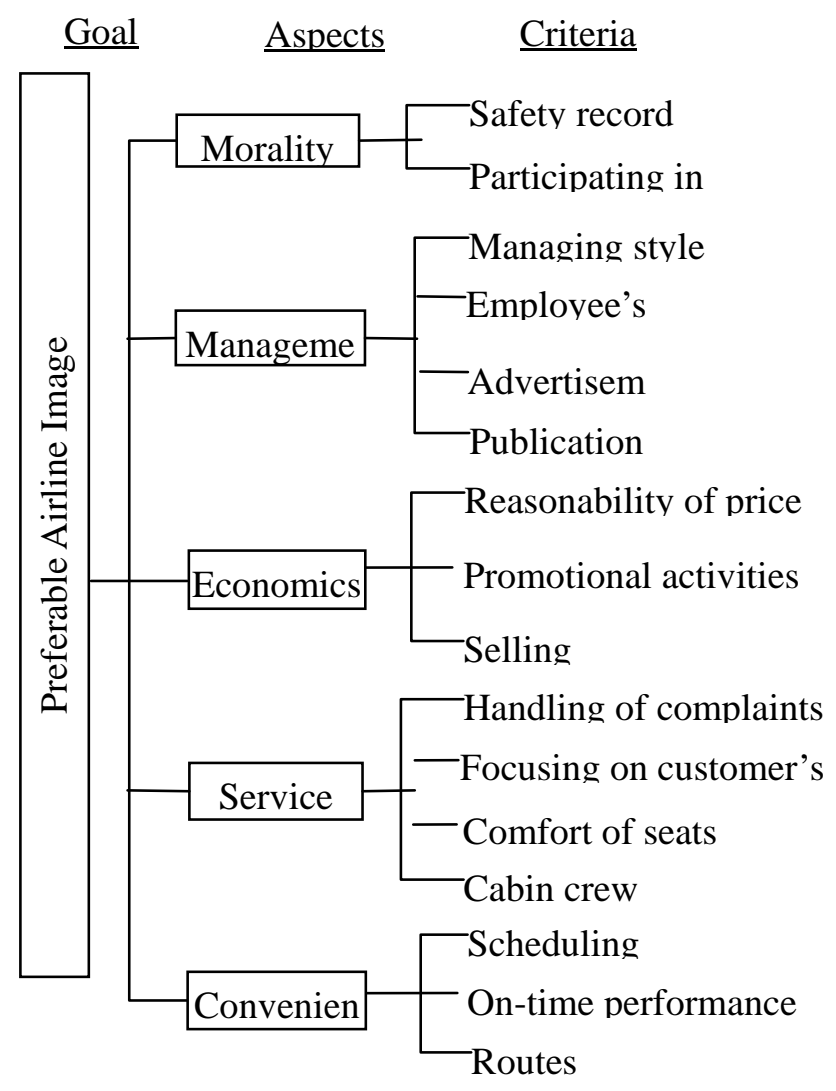

Figure 2. Evaluating Hierarchy For Airline Image

\subsection{Weighting the Evaluated Criteria}

After formulating the hierarchical system for corporate image, we weighted the aspects and criteria. Three groups of evaluators, tour leaders, managers from airline public-relations departments, and passengers from a variety of backgrounds, were involved in the fuzzy AHP weighting process. They 
were requested, via a questionnaire, to compare the relative importance of pair-wise criteria. The application of the fuzzy numbers defined in Table 1 helped to transfer the linguistic scales to the corresponding fuzzy number. Using Eqs. (3), (5), and (7), we obtained the integrated fuzzy weights of the various aspects and criteria for the evaluation system, as shown in Table 2.

Table 2. Fuzzy Weights Of Aspects And Criteria

\begin{tabular}{lccc}
\hline \multicolumn{1}{c}{ Dimension and criteria } & Local weights & Overall weights & BNP \\
\hline Morality & $(0.097,0.221,0.701)$ & & 0.340 \\
Safety record & $(0.036,0.129,0.757)$ & $(0.003,0.028,0.531)$ & 0.188 \\
Participating in charity & $(0.021,0.093,0.480)$ & $(0.002,0.020,0.336)$ & 0.120 \\
Management & $(0.082,0.196,0.595)$ & & 0.291 \\
Airline managing style & $(0.009,0.039,0.396)$ & $(0.001,0.008,0.235)$ & 0.081 \\
Employee's professional skill & $(0.008,0.043,0.342)$ & $(0.001,0.008,0.203)$ & 0.071 \\
Teamwork & $(0.008,0.053,0.366)$ & $(0.001,0.010,0.218)$ & 0.076 \\
Training & $(0.008,0.062,0.385)$ & $(0.001,0.012,0.229)$ & 0.081 \\
Economics & $(0.065,0.189,0.487)$ & & 0.247 \\
Reasonability of price & $(0.012,0.064,0.382)$ & $(0.001,0.012,0.186)$ & 0.066 \\
Promotional activities & $(0.012,0.068,0.370)$ & $(0.001,0.013,0.180)$ & 0.065 \\
Selling channels & $(0.008,0.057,0.274)$ & $(0.001,0.011,0.133)$ & 0.048 \\
Service & $(0.083,0.256,0.588)$ & & 0.309 \\
Handling of complaints & $(0.012,0.067,0.471)$ & $(0.001,0.017,0.277)$ & 0.098 \\
Focusing on customers' interests & $(0.009,0.065,0.375)$ & $(0.001,0.017,0.221)$ & 0.079 \\
Comfort of seats & $(0.007,0.059,0.298)$ & $(0.001,0.015,0.175)$ & 0.064 \\
Cabin crew service & $(0.006,0.065,0.256)$ & $(0.001,0.017,0.151)$ & 0.056 \\
Convenience & $(0.044,0.138,0.337)$ & & 0.173 \\
Scheduling & $(0.008,0.048,0.305)$ & $(0.0004,0.007,0.103)$ & 0.037 \\
On-time performance & $(0.007,0.049,0.255)$, & $(0.0003,0.007,0.086)$ & 0.031 \\
Routes & $(0.005,0.040,0.191)$ & $(0.0002,0.006,0.064)$ & 0.023 \\
\hline
\end{tabular}

\subsection{Determining the Performance Matrix}

A questionnaire was prepared based on the evaluating criteria indicated in Figure 2. The main goal was to evaluate the corporate image of eight airlines. The questionnaire was conducted over a period of two months at the Taoyuan International Airport in Taiwan. To preserve confidentiality, the eight airlines are referred to as $A_{1}, A_{2}, A_{3}, A_{4}, A_{5}, A_{6}, A_{7}$, and $A_{8}$. $A_{3}$ and $A_{4}$ fly from North America and $A_{7}$ from Europe, while the others are from the Asia Pacific area. 446 passengers who had flown on the designated airlines answered the questionnaire. There were at least 50 samples for each airline to ensure that there was no bias toward a particular airline. Since an evaluator's judgment about airline corporate image is inherently vague and subjective, it is very difficult to express such a complex judgment by conventional quantification methods (such as a scale from 1 to 10). A linguistic variable is necessary in these situations. Respondents were asked to rate their satisfaction with the airline for the evaluated criteria using the five linguistic variables 'very poor,' 'poor,' 'fair,' 'good,' and 'very 
good.' The linguistic variables were then transferred to the corresponding triangular fuzzy number, as indicated in Figure 1 (e.g. fuzzy numbers $(10,25,40)$ represent 'poor'). The value scale ranged from $0-100$, with larger scale values denoting that the designated airline had a better image with respect to the criterion. At this point we applied Eq. (8)-(11) to calculate the integrated performance matrix. The performance values of each airline image toward each criterion, before taking the weighting factor into account, are shown in Table 3.

Table 3. Average Fuzzy Performance Values For Each Criterion

\begin{tabular}{|c|c|c|c|c|}
\hline n & $\mathrm{A}_{1}$ & $\mathrm{~A}_{2}$ & $\mathrm{~A}_{3}$ & $\mathrm{~A}_{4}$ \\
\hline Safety record & $(31.5,48.6,65)$ & $1,78.1,89.6)$ & $(48,65.7,80.6)$ & 87 \\
\hline articipating in & $(47.7,65,80.5)$ & $60.9,78,89.8)$ & $(52.4,70,83.9)$ & $(49.8,67.1,81.9)$ \\
\hline le & 47, & 8) & $5.7,7$ & $(50$. \\
\hline nal & $(48$. & 7) & 3 ) & $(46$ \\
\hline am & $(47.7,6$ & $(54,71.4,8$ & $(52.2,69.6,83)$ & $(49.1,66$ \\
\hline 118 & $(45$, & 75. & .6) & $(47.2$ \\
\hline rice & $(44,61.8$ & $(39.8,58$, & $(52.5,69.1,84)$ & $(44.6,6$ \\
\hline es & $(45.6)-1$ & $(39.3,5$ & $(54,71$ & $(42$. \\
\hline & (51 & & 7) & $(42$ \\
\hline ints & $(36.7$ & $(48$ & $(48.6,6$ & $(47.4,63$ \\
\hline 5 on $c$ & $(43.0,6$ & $(49,65$. & $(43.9, \epsilon$ & $(46.5,63$ \\
\hline & $(44.5$ & & $\operatorname{css} 0^{\circ}$ & $(42.8, \epsilon$ \\
\hline abi & $(50.5$, & $(57.9,7$ & $(46.5, \epsilon$ & $(46$. \\
\hline & & & & $(45$ \\
\hline & $(44.6$ & $(53$ & $(45,61$ & $(46.9,63$ \\
\hline & $(53,70.6$, & $(49.6,67$ & $(56.3,72$ & $(45.7,63$. \\
\hline & $\mathrm{A}_{5}$ & $\mathrm{~A}_{6}$ & $\mathrm{~A}_{7}$ & $A_{8}$ \\
\hline fety & 0.8, 78.8, & $(60,76.3,8$ & $(59.2,76$ & $(48.0,6$ \\
\hline ty & $(62$, & $(60$ & $(5$ & $(46$ \\
\hline irlir & $(56,73$. & $(57.9,7$ & $(58.8,7$ & $(48.5,6$ \\
\hline onal & $(56$ & $(5$ & $(5$ & $(45.4,6$ \\
\hline & (58, & $(5$ & $(56.8,7$ & $(45.3,62$ \\
\hline $\lg$ & $(58$ & $(56$ & $(56.4,7$ & $(43.4,6$ \\
\hline & $(40$ & $(36.3,5$ & $(52.6,6$ & $(36.1,53$ \\
\hline ies & $(36.2$ & 49. & $(49,67$. & $(33.2,51.3,69)$ \\
\hline & $(43$. & $(39.6,57$ & $(48.6,66$ & $(41.4,59.3,74$ \\
\hline an & $(47.5$ & (54.6, 7 & $(51$ & $(39.7,5 \varepsilon$ \\
\hline 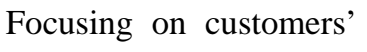 & $(47.5, \epsilon$ & $(4$ & $(4 \varepsilon$ & $(43$. \\
\hline on & $(58,75.5,87.9)$ & $(47.0,64.5,79)$ & $(50.2,69,82.2)$ & $(45.3,62.7,78.8)$ \\
\hline & $(6$ & & $(54$ & \\
\hline & $(50.8,6$ & $(51.9,69, \varepsilon$ & $(48,66,8$ & $(45.4,62.7,79.7)$ \\
\hline On-time performance & $(53.8,71.2,85)$ & $(50.2,68,82.6)$ & $(48.8,67,81.2)$ & $(46.1,63.6,79.7)$ \\
\hline outoc & $(47,64.6,81.1)$ & $(39.1,5$ & $(47.6,66,80.8)$ & $(35.6,53.8,71$ \\
\hline
\end{tabular}

\subsection{Calculating the Final Synthetic Utilities for the Investigated Airlines}

From the criteria weights (Table 2) and the average fuzzy performance values for each criterion (Table 
3), the final fuzzy synthetic utilities can be processed using Eqs. (12), (13). After the fuzzy synthetic decision is processed, the BNP defuzzification method Eq. (14) is then employed. The fuzzy numbers are finally changed into non-fuzzy values. Table 4 shows the aggregated performance values for each airline. A general overview shows that airline $\mathrm{A}_{2}$ performs better than the other airlines. We now discuss the managing of airline image and reputation in-depth.

Table 4. BNP Values For Fuzzy Performance And Ranking

\begin{tabular}{cccc}
\hline Airline & $R_{\mathrm{i}}$ & $B N P_{i}$ & Ranking \\
\hline $\mathrm{A}_{1}$ & $(0.48,3.86,28.55)$ & 29.68 & 8 \\
$\mathrm{~A}_{2}$ & $(0.59,4.39,31.15)$ & 32.42 & 1 \\
$\mathrm{~A}_{3}$ & $(0.56,4.22,30.26)$ & 31.48 & 5 \\
$\mathrm{~A}_{4}$ & $(0.52,4.00,29.33)$ & 30.49 & 6 \\
$\mathrm{~A}_{5}$ & $(0.59,4.41,31.04)$ & 32.32 & 2 \\
$\mathrm{~A}_{6}$ & $(0.58,4.30,30.65)$ & 31.89 & 4 \\
$\mathrm{~A}_{7}$ & $(0.60,4.45,31.00)$ & 32.29 & 3 \\
$\mathrm{~A}_{8}$ & $(0.48,3.83,28.71)$ & 29.82 & 7 \\
\hline
\end{tabular}

\section{Discussion}

In this empirical study, we develop a quantitative system to measure airline image. We extract 16 criteria which are then used to construct a hierarchal system, where five aspects related to airline image and reputation are evaluated. Table 2 shows the relative weights of these five aspects of airline image, as obtained from fuzzy AHP. The results in Table 2 show that morality $(0.34)$ and service $(0.30)$ are the two most important aspects, while the least important is convenience. Apparently, morality and service are most important to airline image. This suggests that airlines should concentrate on these two factors as the foundation of a good reputation. It can also be seen that the most important criterion within the morality category is the safety record. This is because the public focuses more attention on accidents than other types of news related to an airline. In other words, airlines need to maintain a good safety record to keep an excellent reputation; safety is the key for a successful business in the aviation industry. In the service category, the handling of complaints plays an important role in customer perception. This suggests that air carriers can improve their reputation by carefully handling complaints and striving to meet their customers' needs. Furthermore airlines should educate their employee about how to deal with complaints. In contrast, convenience and incentives seem to have little attraction for passengers.

The final aggregated reputation index for each airline, based on the proposed fuzzy MCDM model and computed using the SAW method, is shown in Table 4. If we define $\mathrm{A} \succ \mathrm{B}$ this means that $\mathrm{A}$ outranks $B$; the ranking of the reputation index (image level) for the surveyed airlines is as follows: $A_{2}$ $\succ \mathrm{A}_{5} \succ \mathrm{A}_{7} \succ \mathrm{A}_{6} \succ \mathrm{A}_{3} \succ \mathrm{A}_{4} \succ \mathrm{A}_{8} \succ \mathrm{A}_{1}$. It is noted that the airlines can be divided into 
three groups, the leading group, $A_{2}, A_{5}$, and $A_{7}$; the competitive group, $A_{3}, A_{4}$, and $A_{6}$; and the lagging group, $A_{1}$ and $A_{8}$. As can be seen from the performance matrix (Table 3 ), the leading airlines have excellent performance in terms of both safety and service, which are major factors in influencing the reputation of airlines. Although $\mathrm{A}_{2}$ is temporarily in the leading position, it needs to lower its price to keep its leading status in reputation. $A_{5}$ has some room to improve in terms of its promotional program. In the competitive group, the performance of $A_{3}, A_{4}$, and $A_{6}$ is very close. If $A_{3}$ could invest in improving the comfort of its seats, or $\mathrm{A}_{4}$ their incentive program, they might possibly move to a leading position. In the lagging group, $\mathrm{A}_{1}$ and $\mathrm{A}_{8}$ received poor grades in many areas, meaning they need to improve every aspect to catch up with the other airlines. Though $A_{1}$ has a good image in terms of cabin crew service, their safety record was rated particularly poor by customers, meaning they need to improve their safety program in order to elevate their reputation. $A_{8}$ had an obviously poor image in terms of handling customer complaints, so probably needs to strengthen their customer relation management (CRM).

The results show that the safety record is most important to airline image and reputation in Taiwan. This is not difficult to understand, since the less than desirable safety record of Asian airlines in general has given air travelers in Taiwan cause for concern. Therefore, the key to a successful strategy is to rebuild the confidence of air transport customers. Although the selected criteria are strongly influenced by the local environment and culture, the proposed factor structure can easily be transformed and extended to the conditions and culture in other markets in any particular environment.

\section{Conclusions}

Corporate image is particularly important in the service industry and can heavily influence customers' buying behaviors. However, corporate image is an unfamiliar, intangible, subjective, transitory, and vague concept that is difficult to measure. With the help of the fuzzy AHP and FMCDM, however, this abstract concept can be quantitatively measured. This measurement can help managers develop strategies for enhancing their firm's position. A hybrid fuzzy MCDM model is proposed. First some criteria for corporate image are identified through a literature review and survey. Second, we apply the fuzzy AHP to determine the relative weights for each independent common factor. Finally, the FMCDM method is conducted to evaluate the corporate image and reputation. The constructed hybrid fuzzy MCDM model is an effective way to measure corporate image and to help a company understand its relative level of reputation. Consequently, managers can fully comprehend the relative positions of their firm in the market, make better informed judgments, and act decisively. Additionally, it can improve the gaps between real performance and the desired/aspiration level to meet customer expectations.

\section{REFERENCES}

Barich, H. and Kotler, P. (1991) A framework for marketing image management, Sloan Management 
Review, 32(2), 94-104.

Bellman, R.E. and Zadeh, L.A. (1970) Decision-making in a fuzzy environment, Management Science, 17(4), 141-164.

Buckley, J,J. (1985a) Fuzzy hierarchical analysis, Fuzzy Sets Systems, 17(1), 233-247.

Buckley, J.J. (1985b) Ranking alternatives using fuzzy numbers, Fuzzy Sets Systems, 15(1), 21-31.

Campbell, A. (1994) Verbeke A. For a strategic view of managing multinationals in hotels industry, Long Range Planning, 27(2), 95-102.

Chiou, H.K., Tzeng, G.H. and Cheng, D.C. (2005) Evaluating sustainable fishing development strategies using fuzzy MCDM approach, OMEGA, 33(3), 223-234.

Dichter, E. (1985) What's in an image? Journal of Consumer Marketing, 2(1), 75-81.

Dubis, D. and Prade, H. (1978) Operations on fuzzy numbers. International Journal of Systems Science, 9(7), 613-626.

Gray, E. and Balmer, J. (1998) Managing corporate image and corporate reputation. Long Range Planning, 31(5), 695-702.

Hwang, C.L. and Yoon, K. (1981) Multiple attribute decision making, lecture notes in economics and mathematical systems. Berlin: Springer.

Hsieh, T.Y., Lu, S.T. and Tzeng, G.H. (2004) Fuzzy MCDM approach for planning and design tenders selection in public buildings, International Journal of Project Management, 22(7), 573-584.

Hsu, Y.G., Shyu, J.Z. and Tzeng, G.H. (2005) Policy tools on the formation of new biotechnology firms in Taiwan, Technovation, 25(3), 281-292.

Keeney, R.L. and Raiffa, H. (1976) Decisions with multiple objectives: preference and value tradeoffs, New York: Wiley.

Mon, D.L., Cheng, C.H. and Lin, J.C. (1994) Evaluation weapon using fuzzy analytic hierarchy process based on entropy weight, Fuzzy Sets Systems, 62(2), 127-134. 
Nguyen, N. and Leblanc, G. (2001) Corporate image and corporate reputation in customers' retention decisions in services, Journal of Retailing and Consumer Services, 8(4), 227-236.

Rao, H. (1994) The social construction of reputation: certification contests, legitimation, and the survival of organizations in the American automobile industry: 1895-1912, Strategic Management Journal, 15(1), 29-44.

Ruyter, K. and Wetzels, M. (2000) The role of corporate image and extension similarity in service brand extensions, Journal of Economic Psychology, 21(16), 639-659.

Saaty. T. (1980) The analytic hierarchy process, New York: McGraw-Hill.

Saaty T. (1977) A scaling method for priorities in hierarchical structures. Journal of Mathematical Psychology, 15(3), 234-281.

Schmitt, B., Simonson, A. and Marcus, J. (1995) Managing corporate image and identity, Long Range Planning, 28(5), 82-92.

Teng, J.Y. and Tzeng, G.H. (1996) Fuzzy multicriteria ranking of urban transportation investment alternative, Transportation Planning and Technology, 20(1), 15-31.

Williams, S.L. and Moffitt, M.A. (1997) Corporate image as an impression formation process: Prioritizing Personal, Organizational, and Environmental Audience Factors, Journal of Public Relations Research, 9(4), 237-258.

Zadeh, L.A. (1965) Fuzzy sets. Information and Control, 8(3), 338-353.

Zadeh, L.A. (1975) The concept of a linguistic variable and its application to approximate reasoning, Information Science, 8(1), pp.199-249; 8(2), 301-357.

Zhao, R. and Goving, R. (1991) Algebraic characteristics of extended fuzzy numbers. Information Science, 54(1-2), 103-130. 